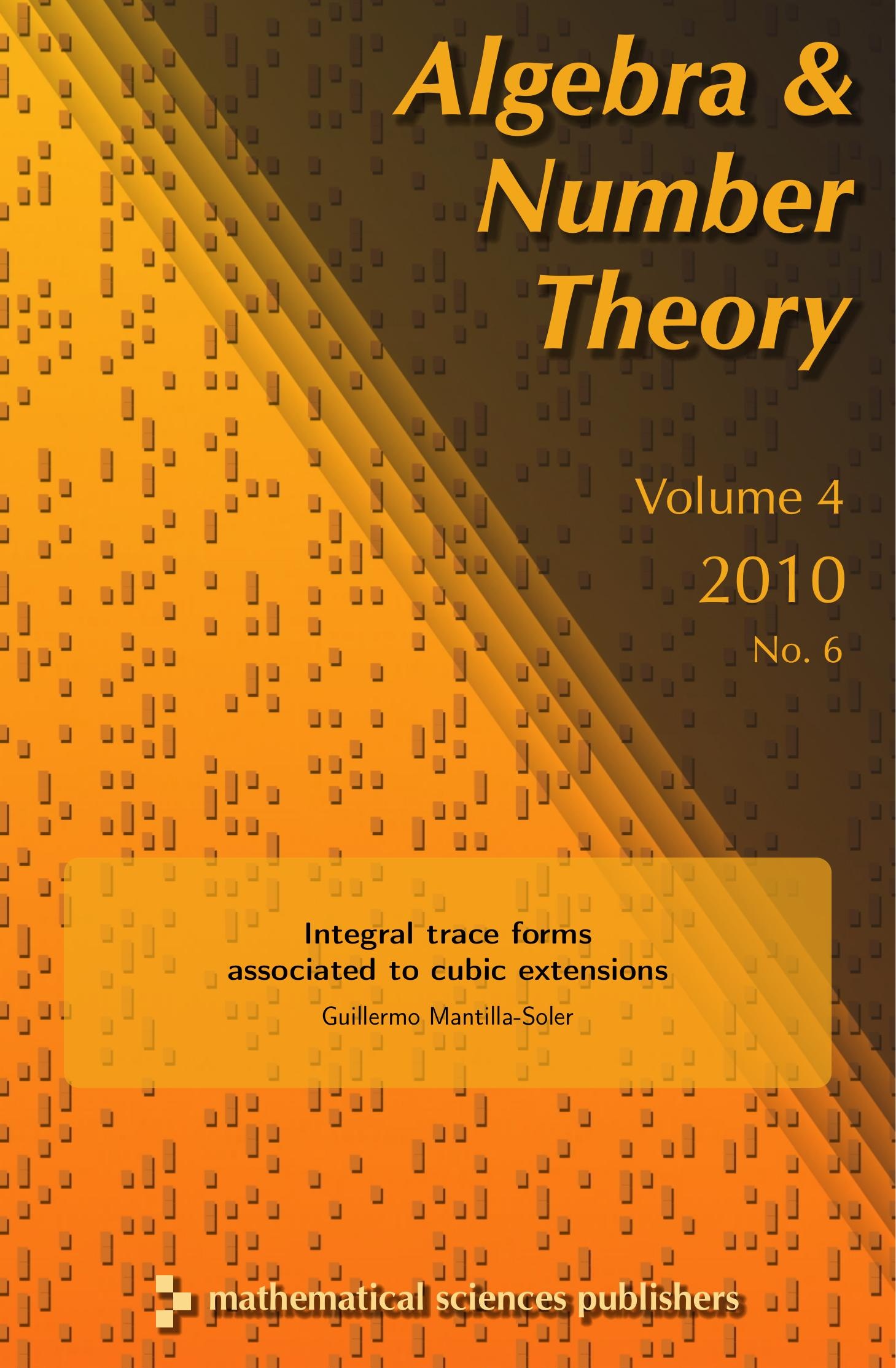




\title{
Integral trace forms associated to cubic extensions
}

\author{
Guillermo Mantilla-Soler
}

Given a nonzero integer $d$, we know by Hermite's Theorem that there exist only finitely many cubic number fields of discriminant $d$. However, it can happen that two nonisomorphic cubic fields have the same discriminant. It is thus natural to ask whether there are natural refinements of the discriminant which completely determine the isomorphism class of the cubic field. Here we consider the trace form $q_{K}:\left.\operatorname{tr}_{K / \mathbb{Q}}\left(x^{2}\right)\right|_{O_{K}^{0}}$ as such a refinement. For a cubic field of fundamental discriminant $d$ we show the existence of an element $T_{K}$ in Bhargava's class group $\mathrm{Cl}\left(\mathbb{Z}^{2} \otimes \mathbb{Z}^{2} \otimes \mathbb{Z}^{2} ;-3 d\right)$ such that $q_{K}$ is completely determined by $T_{K}$. By using one of Bhargava's composition laws, we show that $q_{K}$ is a complete invariant whenever $K$ is totally real and of fundamental discriminant.

1. Introduction

2. Basic facts

3. Galois fields and rational 3-torsion

4. Cubic fields with fundamental discriminant

5. Trace form and class groups

6. From cubic fields to cubes and trace forms

694

Acknowledgements

698

References

698

\section{Introduction}

Generalities. A difference between quadratic and nonquadratic number fields is that the former are totally characterized by their discriminant. One natural choice for a refined discriminant is given by the isometry class with respect to the trace form of the lattice defined by the maximal order. The purpose of this paper is to give a detailed analysis of this refinement for cubic extensions, and to show under which conditions this refinement characterizes the field. Given a number field $K$ with maximal order $O_{K}$, we consider the trace form $\left.\operatorname{tr}_{K / \mathbb{Q}}\left(x^{2}\right)\right|_{O_{K}}$.

MSC2000: primary 11E12; secondary 11R29, 11R16, 11E76.

Keywords: integral trace forms, cubic fields, Bhargava's class group, discriminants of number fields. 
Question 1.1. Do there exist two nonisomorphic number fields $K$ and $L$ such that their corresponding trace forms are isomorphic?

In this paper we analyze this question in the case of cubic extensions.

Definition 1.2. Let $K$ be a number field and let $O_{K}$ be its maximal order. The trace zero module $O_{K}^{0}$ is the set $\left\{x \in O_{K}: \operatorname{tr}_{K / \mathbb{Q}}(x)=0\right\}$.

Our main result is this:

Theorem 6.5. Let $K$ be a cubic number field of positive, fundamental discriminant. Let $L$ be a number field such that there exists an isomorphism of quadratic modules

$$
\left\langle O_{K}^{0},\left.\operatorname{tr}_{K / \mathbb{Q}}\left(x^{2}\right)\right|_{O_{K}^{0}}\right\rangle \cong\left\langle O_{L}^{0},\left.\operatorname{tr}_{L / \mathbb{Q}}\left(x^{2}\right)\right|_{O_{L}^{0}}\right\rangle,
$$

and assume $9 \nmid d_{L}$. Then $K \cong L$.

Outline of the paper. We start by analyzing Question 1.1 for general cubic fields. For this purpose we consider first the case in which the common discriminant of $K$ and $L$ is not fundamental. ${ }^{1}$

Nonfundamental discriminants. In this case, we find that our proposed refinement does not characterize the field. In other words, for nonfundamental discriminants we have an affirmative answer to Question 1.1. We divide the class of nonfundamental discriminants into two groups according to sign. We further divide the positive discriminants into two groups: those that are perfect squares, and those that are not. For each one of these cases we show that there are some nonfundamental discriminants such that Question 1.1 has an affirmative answer.

(i) Negative nonfundamental discriminants. We define a sequence of positive integers $\Sigma$ and a family of triples $\left\{K_{m}, L_{m}, E_{m}\right\}_{m \in \Sigma}$ with the following properties (see Proposition 3.4):

- $K_{m}$ and $L_{m}$ are two nonisomorphic cubic fields with discriminant $-3 n^{2}$, where $n$ is a positive integer depending only on $m$.

- An elliptic curve $E_{m}$ defined over $\mathbb{Q}$ such that $E_{m}[3](\mathbb{Q})$ determines completely a ternary quadratic form equivalent to both $\left.\operatorname{tr}_{K / \mathbb{Q}}\left(x^{2}\right)\right|_{O_{K_{m}}}$ and $\left.\operatorname{tr}_{K / \mathbb{Q}}\left(x^{2}\right)\right|_{O_{m}}$.

(ii) Square discriminants. In this case we generalize in Theorem 3.1 a result of Conner and Perlis [1984, Theorem IV.1.1 with $p=3$ ]. Let $K$ and $L$ be two Galois cubic number fields of the same discriminant and let $M$ be either $O_{K}$ or $O_{K}^{0}$. Then $\left.\operatorname{tr}_{K / \mathbb{Q}}\left(x^{2}\right)\right|_{M}$ and $\left.\operatorname{tr}_{L / \mathbb{Q}}\left(x^{2}\right)\right|_{M}$ are equivalent. Since there are examples of nonisomorphic Galois cubic fields of the same discriminant, Question 1.1 has a positive answer for such cases.

\footnotetext{
${ }^{1}$ Recall that $d$ is a fundamental discriminant if it is the discriminant of a quadratic field.
} 
(iii) Positive, nonfundamental, nonsquare discriminants. See Example 3.6 for two fields with positive, non-square-free, non-perfect-square discriminant and isometric integral trace forms.

Main results. For fields of fundamental discriminant we see, thanks to Lemma 2.5, that the binary quadratic form $\left.\operatorname{tr}_{K / \mathbb{Q}}\left(x^{2}\right)\right|_{O_{K}^{0}}$ is a refinement of the discriminant. Hence, we reformulate Question 1.1.

Question 1.3. Do there exist two nonisomorphic cubic fields $K$ and $L$ such that the forms $\left.\operatorname{tr}_{K / \mathbb{Q}}\left(x^{2}\right)\right|_{O_{K}^{0}}$ and $\left.\operatorname{tr}_{L / \mathbb{Q}}\left(x^{2}\right)\right|_{O_{L}^{0}}$ are isomorphic?

Although this question has relevance for us only for fundamental discriminants, we note that the examples (i), (ii) and (iii) described above also answer 1.3 in an affirmative way. On the other hand, for fundamental discriminants (see diagram 4-1), class field theory provides examples of nonisomorphic cubic fields of the same discriminant. Among the fields with negative discriminants we found examples giving an affirmative answer to Question 1.3.

It is clear, thanks to the results developed so far, that one should consider working over cubic fields of fundamental discriminant. We show for such discriminants that the trace form is equal, as an element of a narrow class group, to the Hessian multiplied by an element that only depends on the discriminant.

Theorem 5.5. Let $K$ be a cubic field with discriminant $d_{K}$. Assume that $d_{K}$ is fundamental and that $3 \nmid d_{K}$. Let $F_{K}=(a, b, c, d)$ be a cubic in the $\mathrm{GL}_{2}(\mathbb{Z})$ equivalence class defined by $K$. Then $\frac{1}{2} q_{K} * C_{d_{K}}=H_{K}^{ \pm 1}$ as elements of $\mathrm{Cl}_{\mathbb{Q}\left(\sqrt{-3 d_{K}}\right)}^{+}$, where $C_{d_{K}}=\left(3,0, d_{K} / 4\right)$ or $C_{d_{K}}=\left(3,3,\left(d_{K}+3\right) / 4\right)$ in accordance with whether $d_{k} \equiv 0(\bmod 4)$ or $d_{k} \equiv 1(\bmod 4)$.

By reformulating all of this in the language of Bhargava's composition of cubes [2004], we show that the trace form arises naturally as a projection of a cube determined by the field.

Theorem 6.2. Let $K$ be a cubic field with discriminant $d_{K}$ and associated cubic form $F_{K}=(a, b, c, d)$. Assume that $d_{K}$ is fundamental and that 3 does not ramify. Then there exists $T_{F_{K}} \in \mathrm{Cl}\left(\mathbb{Z}^{2} \otimes \mathbb{Z}^{2} \otimes \mathbb{Z}^{2} ;-3 d_{K}\right)$ such that $\left(\pi_{1} \circ \phi\right)\left(T_{F_{K}}\right)^{ \pm 1}=\frac{1}{2} q_{K}$ as elements of $\mathrm{Cl}_{\mathbb{Q}\left(\sqrt{-3 d_{K}}\right)}^{+}$.

In this setting, Theorem 6.5 follows from Theorem 5.11, which is the modern version of a theorem of Eisenstein [1844]. By reformulating Theorem 6.5 (see Theorem 6.8 and its corollary), we obtain one inequality of the classical Scholz reflection principle [1932].

Theorem 6.5 can be obtained with the tools developed by Eisenstein [1844]. However, we have decided to use Bhargava's theory of $2 \times 2 \times 2$ orbits of cubes, to suggest that it might be possible to use some other prehomogeneous spaces to "generalize" Theorem 6.5 to higher dimensions. 


\section{Basic facts}

Definition 2.1. Let $G$ be a free abelian group. We say that a map $q: G \rightarrow \mathbb{Z}$ is a quadratic form if

- $q(n x)=n^{2} q(x)$ for all integer $n$, and

- the map $B_{q}: G \times G \rightarrow \frac{1}{2} \mathbb{Z}$ defined as $B_{q}(x, y)=\frac{1}{2}(q(x+y)-q(x)-q(y))$ is $\mathbb{Z}$-bilinear.

Remark 2.2. Let $\langle G, q\rangle$ be a quadratic $\mathbb{Z}$-module of $\operatorname{rank} n=\operatorname{rank}(G)$. After choosing a basis, we can think of $q$ as a homogeneous polynomial in $n$ variables of degree two, that is, $q \in\left(\mathrm{Sym}^{2} \mathbb{Z}^{n}\right)^{*}$. There is a natural action of $\mathrm{GL}_{2}(\mathbb{Z})$ on $\left(\operatorname{Sym}^{2} \mathbb{Z}^{n}\right)^{*}$. Under this action, $q$ and $q_{1}$ belong to the same orbit if and only if $\langle G, q\rangle$ is isometric to $\left\langle G_{1}, q_{1}\right\rangle$. Abusing notation, we denote this by $q \sim_{\mathrm{GL}_{2}(\mathbb{Z})} q_{1}$.

Let $K$ be a number field and let $O_{K}$ be its maximal order. The map

$$
\tilde{q}_{K}: O_{K} \rightarrow \mathbb{Z}, \quad x \mapsto \operatorname{tr}_{K / \mathbb{Q}}\left(x^{2}\right),
$$

defines a quadratic form with corresponding bilinear form

$$
B_{K}(x, y)=\operatorname{tr}_{K / \mathbb{Q}}(x y) \mid o_{K} .
$$

Thus, $\left\langle O_{K}, \tilde{q}_{K}\right\rangle$ is a quadratic $\mathbb{Z}$-module and its discriminant is precisely the discriminant of $K$. Hence, if $K$ and $L$ are two number fields such that $\left\langle O_{K}, \tilde{q}_{K}\right\rangle$ and $\left\langle O_{L}, \tilde{q}_{L}\right\rangle$ are isomorphic quadratic $\mathbb{Z}$-modules, we have

$$
[K: \mathbb{Q}]=[L: \mathbb{Q}] \text { and } \operatorname{Disc} K=\operatorname{Disc} L .
$$

Therefore the isomorphism class of $\left\langle O_{K}, \tilde{q}_{K}\right\rangle$ is to us a natural refinement of the discriminant.

Lemma 2.3. Let $K$ be a number field of degree $n$ and let $G_{K}=\mathbb{Z}+O_{K}^{0}$. We have

$$
\left|O_{K} / G_{K}\right|=\left|\operatorname{tr}_{K / \mathbb{Q}}\left(O_{K}\right) / n Z\right| .
$$

Corollary 2.4. Let $K$ and $L$ be number fields. If

$$
f:\left\langle O_{K}, B_{K}\right\rangle \rightarrow\left\langle O_{L}, B_{L}\right\rangle
$$

is an isomorphism, then $\operatorname{Disc} G_{K}=\operatorname{Disc} G_{L}$.

Proof. Since $\operatorname{tr}_{L / \mathbb{Q}}(f(x) f(y))=\operatorname{tr}_{K / \mathbb{Q}}(x y)$ for all $x, y \in O_{K}$ we have that $\operatorname{tr}_{K / \mathbb{Q}}$ : $O_{K} \rightarrow \mathbb{Z}$ implies $\operatorname{tr}_{L / \mathbb{Q}}: O_{L} \rightarrow \mathbb{Z}$. Since $f$ is an isometry, the argument is symmetric in $K$ and $L$. By Lemma 2.3 we have $\left|O_{K} / G_{K}\right|=\left|O_{L} / G_{L}\right|$. Hence

$\operatorname{Disc} G_{K}=\left|O_{K} / G_{K}\right|^{2} \operatorname{Disc} O_{K}=\left|O_{L} / G_{L}\right|^{2} \operatorname{Disc} O_{L}=\operatorname{Disc} G_{L}$.

For a number field $K$, we set $q_{k}=\left.\tilde{q}_{K}\right|_{O_{K}^{0}}$. 
Lemma 2.5. Let $K$ and $L$ be two number fields of degree $n$. Assume they both have discriminants that are square-free at all primes dividing $n$. Further, suppose that $\left\langle O_{K}^{0}, q_{K}\right\rangle$ and $\left\langle O_{L}^{0}, q_{L}\right\rangle$ are isomorphic. Then $K$ and $L$ have the same discriminant.

Proof. Since Disc $G_{K}=\operatorname{Disc} G_{L}$, we have that

$$
\left|O_{K} / G_{K}\right|^{2} \operatorname{Disc} O_{K}=\left|O_{L} / G_{L}\right|^{2} \operatorname{Disc} O_{L} \text {. }
$$

The result now follows from Lemma 2.3.

Proposition 2.6. Let $K$ be a Galois number field of prime degree $p$. Then $p$ ramifies in $K$ if and only if $\operatorname{tr}_{K / \mathbb{Q}}\left(O_{K}\right)=p \mathbb{Z}$.

Proof. It is clear that $\operatorname{tr}_{K / \mathbb{Q}}\left(O_{K}\right)=p \mathbb{Z}$ implies that $p$ ramifies in $K$. Next, assuming that $p$ ramifies, let $P$ be the unique prime of $O_{K}$ lying above $p$. By hypothesis, we have that $\left|O_{K} / P\right|=p$. In particular, $P$ is a maximal $\mathbb{Z}$-submodule of $O_{K}$. Since $1 \notin P$, we must have $O_{K}=\mathbb{Z}+P$. Since $P$ is Galois invariant, $\operatorname{tr}_{K / \mathbb{Q}}(P) \subseteq$ $P \cap \mathbb{Z}=p \mathbb{Z}$. Thus $\operatorname{tr}_{K / \mathbb{Q}}\left(O_{K}\right)=\operatorname{tr}_{K / \mathbb{Q}}(\mathbb{Z}+P) \subseteq p \mathbb{Z}$.

\section{Galois fields and rational 3-torsion}

In this section we explain some situations in which Questions 1.1 and 1.3 have positive answers. The examples in this section are characterized by having discriminants with a nontrivial square factor.

The following result is a generalization of the case $p=3$ of [Conner and Perlis 1984, Theorem IV.1.1].

Theorem 3.1. Let $K$ and $L$ be two Galois, cubic number fields of discriminant $D=d^{2}$. We have

$$
\left\langle O_{K}^{0}, q_{K}\right\rangle \cong\left\langle O_{L}^{0}, q_{L}\right\rangle \cong \begin{cases}2 d\left(x^{2}+x y+y^{2}\right) & \text { if } 3 \nmid d, \\ \frac{2}{3} d\left(x^{2}+x y+y^{2}\right) & \text { otherwise. }\end{cases}
$$

The isometry can be chosen so it extends to one between $\left\langle O_{K}, \tilde{q}_{K}\right\rangle$ and $\left\langle O_{L}, \tilde{q}_{L}\right\rangle$.

Proof. Assume first that 3 does not divide $D$. By Theorem 132 of Hilbert [1900], write $O_{K}=e_{1} \mathbb{Z} \oplus e_{2} \mathbb{Z} \oplus e_{3} \mathbb{Z}$, where $\sigma\left(e_{1}\right)=e_{2}, \sigma\left(e_{2}\right)=e_{3}$, and $\sigma$ is a generator of $\operatorname{Gal}(K / \mathbb{Q})$. Because 3 does not ramify, Proposition 2.6 implies that $\operatorname{tr}_{F / \mathbb{Q}}\left(e_{1}\right)=1$, and furthermore that $O_{K}^{0}=\left(e_{1}-e_{2}\right) \mathbb{Z} \oplus\left(e_{1}-e_{3}\right) \mathbb{Z}$. Let $a=\operatorname{tr}_{F / \mathbb{Q}}\left(e_{1}^{2}\right)$ and $b=$ $\operatorname{tr}_{F / \mathbb{Q}}\left(e_{1} e_{2}\right)$. Then

$$
M=\left(\begin{array}{ccc}
(1+2 a-2 b) / 3 & a-b & a-b \\
a-b & 2 a-2 b & a-b \\
a-b & a-b & 2 a-2 b
\end{array}\right) \quad \text { and } \quad M_{0}=(a-b)\left(\begin{array}{ll}
2 & 1 \\
1 & 2
\end{array}\right)
$$

represent respecitvely the trace form over $O_{K}$ in the basis $\left\{e_{1}, e_{1}-e_{2}, e_{1}-e_{3}\right\}$, and the trace form over $O_{K}^{0}$ in the basis $\left\{e_{1}-e_{2}, e_{2}-e_{3}\right\}$. Note that $a+2 b=$ 
$\left(\operatorname{tr}_{F / \mathbb{Q}}\left(e_{1}\right)\right)^{2}=1$; thus $D=\operatorname{det} M=(a-b)^{2}(a+2 b)=(a-b)^{2}$. By the CauchySchwartz inequality, $a-b>0$, and hence $d=a-b$, which implies that $a=$ $(1+2 d) / 3$ and $b=(1-d) / 3$. Thus, every cubic field of discriminant $d^{2}$, with $3 \nmid d$, has an integral basis for which the trace form over $O_{K}$ has representative matrix $M$ (respectively trace form over $O_{K}^{0}$ has representative matrix $M_{0}$ ).

On the other hand, if 3 divides $d$, then Proposition 2.6 and Lemma 2.3 imply that $O_{K}=\mathbb{Z} \oplus O_{K}^{0}$. Hence, $\tilde{q}_{K}$ is totally determined by $q_{K}=\left.\tilde{q}_{K}\right|_{O_{K}^{0}}$. Since every integral quadratic form of discriminant -3 is $\mathrm{SL}_{2}(\mathbb{Z})$-equivalent to $\left(x^{2}+x y+y^{2}\right)$, the result follows from the following claim.

Claim. $\frac{3}{2 d} q_{K}$ is an integral, primitive, binary quadratic form of discriminant -3 .

Proof of claim. Let $\{\alpha, \beta\}$ an integral basis for $O_{K}^{0}$. Let $O_{\alpha} \subseteq O_{K}^{0}$ be the $\mathbb{Z}$-module generated by $\{\alpha, \sigma(\alpha)\}$, where $\sigma$ is a generator for $\operatorname{Gal}(K / \mathbb{Q})$. Since $\alpha \notin \mathbb{Z}$, we know that $\alpha$ and $\sigma(\alpha)$ are distinct elements of $O_{K}$ with the same norm. In particular, $\sigma(\alpha)$ cannot be a rational multiple of $\alpha$, so $\operatorname{rank}_{\mathbb{Z}}(O)=2$. Thus, $\left[O_{K}^{0}: O_{\alpha}\right]$ is finite, and moreover $\sigma(\alpha)=m \alpha+\left[O_{K}^{0}: O_{\alpha}\right] \beta$ for some integer $m$. Note that $\left(\operatorname{tr}_{K / \mathbb{Q}}\left(\alpha^{2}\right), 2 \operatorname{tr}_{K / \mathbb{Q}}(\alpha \beta), \operatorname{tr}_{K / \mathbb{Q}}\left(\beta^{2}\right)\right),\left(\operatorname{tr}_{K / \mathbb{Q}}\left(\alpha^{2}\right), 2 \operatorname{tr}_{K / \mathbb{Q}}(\alpha \sigma(\alpha)), \operatorname{tr}_{K / \mathbb{Q}}\left(\sigma(\alpha)^{2}\right)\right)$ represent $q_{K}$ in the bases $\{\alpha, \beta\}$ and $\{\alpha, \sigma(\alpha)\}$ respectively. Hence

$$
\begin{aligned}
\operatorname{tr}_{K / \mathbb{Q}}\left(\alpha^{2}\right) \operatorname{tr}_{K / \mathbb{Q}}\left(\sigma(\alpha)^{2}\right) & -\operatorname{tr}_{K / \mathbb{Q}}^{2}(\alpha \sigma(\alpha)) \\
& =\left[O_{K}^{0}: O_{\alpha}\right]^{2}\left(\operatorname{tr}_{K / \mathbb{Q}}\left(\alpha^{2}\right) \operatorname{tr}_{K / \mathbb{Q}}\left(\beta^{2}\right)-\operatorname{tr}_{K / \mathbb{Q}}(\alpha \beta)\right) .
\end{aligned}
$$

Since Disc $K=d^{2}$ and $O_{K}=\mathbb{Z}+O_{K}^{0}, \frac{1}{3} d^{2}=\operatorname{tr}_{K / \mathbb{Q}}\left(\alpha^{2}\right) \operatorname{tr}_{K / \mathbb{Q}}\left(\beta^{2}\right)-\operatorname{tr}_{K / \mathbb{Q}}(\alpha \beta)$. On the other hand, since $\alpha \in O_{K}^{0}, \operatorname{tr}_{K / \mathbb{Q}}\left(\alpha^{2}\right)=-2 \operatorname{tr}_{K / \mathbb{Q}}(\alpha \sigma(\alpha))$, and the left side of (3-1) is $3 \operatorname{tr}_{K / \mathbb{Q}}^{2}(\alpha \sigma(\alpha))$. Thus,

$$
\operatorname{tr}_{K / \mathbb{Q}}(\alpha \sigma(\alpha))= \pm\left[O_{K}^{0}: O_{\alpha}\right] \frac{d}{3} \text {. }
$$

In particular we see that $\frac{d}{3}$ divides $\frac{1}{2} \operatorname{tr}_{K / \mathbb{Q}}\left(\alpha^{2}\right)$. Exchanging the roles of $\alpha$ and $\beta$ we see that $\frac{d}{3}$ also divides $\frac{1}{2} \operatorname{tr}_{K / \mathbb{Q}}\left(\beta^{2}\right)$. Now consider $\sigma(\alpha)=m \alpha+\left[O_{K}^{0}: O_{\alpha}\right] \beta$. Multiplying both sides by $\alpha$ and then taking traces, we see that $\frac{d}{3}$ divides $\operatorname{tr}_{K / \mathbb{Q}}(\alpha \beta)$. We conclude that $\left.\operatorname{tr}_{K / \mathbb{Q}}\left(\alpha^{2}\right), 2 \operatorname{tr}_{K / \mathbb{Q}}(\alpha \beta), \operatorname{tr}_{K / \mathbb{Q}}\left(\beta^{2}\right)\right)$ can be written as $\frac{2 d}{3} f$, with $f$ an integral quadratic form of discriminant -3 .

Example 3.2. Let $K$ and $L$ be cubic fields defined by $x^{3}+6 x^{2}-9 x+1$ and $2 x^{3}+3 x^{2}-9 x+2$ respectively. One sees by direct computation that $K$ and $L$ are nonisomorphic fields of discriminant 3969; for instance, they have different regulators.

We conclude that the trace form does not characterize the field in the case where the discriminant is a square. Proposition 3.4 below is an indication that the case of the square discriminant is not the only case that should be reconsidered, but also the 
non-square-free case. Cubic fields of a fixed discriminant $\Delta$ can be parametrized by a subset of rational points on a certain elliptic curve. Assume that $L=\mathbb{Q}(\beta)$ is a cubic field defined by the equation $x^{3}+p x+q \in \mathbb{Z}[x]$. If $O_{L}=\mathbb{Z}[\beta]$, then Disc $L=$ $-27 q^{2}-4 p^{3}$. Hence if $K$ is a cubic field of discriminant $\Delta$, one could try to find a cubic field $L$ of the same discriminant by finding rational points $\left(-\frac{1}{3} p, \pm \frac{1}{2} q\right)$ of $y^{2}=x^{3}-\frac{1}{108} \Delta$. Using this idea, we construct a family of nonisomorphic cubic fields with prescribed discriminant. We need the following result from algebraic number theory.

Proposition 3.3 [Marcus 1977]. Let $m$ be a nonperfect cube integer and $\alpha$ a root of $x^{3}-m$. Write $m=m_{f} m_{s}^{2}$, with $m_{f}$ square-free and $\operatorname{gcd}\left(m_{f}, m_{s}\right)=1$. Suppose that $m \not \equiv \pm 1(\bmod 9)$. Then $\left\{1, \alpha, \alpha^{2} / m_{s}\right\}$ is an integral basis for $K_{m}=\mathbb{Q}(\alpha)$; in particular, Disc $K_{m}=-27\left(m_{s} m_{f}\right)^{2}$.

Let $\Sigma=\left\{m \in \mathbb{N} \backslash \mathbb{N}^{3} \mid m_{s} \neq 1, m_{f} m_{s} \not \equiv \pm 1(\bmod 9), m \neq \equiv \pm 1(\bmod 9)\right\}$.

Proposition 3.4. Let $m \in \Sigma$ and $K_{m}, L_{m}$ be the cubic fields defined by $x^{3}-m$ and $x^{3}-m_{f} m_{s}$ respectively, with $m_{f}, m_{s}$ as in Proposition 3.3. Then $K_{m}, L_{m}$ are cubic fields with equivalent trace forms, and have discriminant $-3\left(3 m_{f} m_{s}\right)^{2}$.

Proof. By the discussion above and Proposition 3.3, we have that $K_{m}$ defines the rational elliptic curve $E_{m}: y^{2}=x^{3}+\frac{1}{4} m_{f}^{2} m_{s}^{2}$. A simple calculation shows that $E_{m}[3](\mathbb{Q})=\left\{\infty,\left(0, \frac{1}{2} m_{f} m_{s}\right),\left(0,-\frac{1}{2} m_{f} m_{s}\right)\right\}$, and these points define the field $L_{m}$. Let $P$ be a generator of $E_{m}[3](\mathbb{Q})$ and let

$$
M_{m}=\left(\begin{array}{ccc}
3 & 0 & 0 \\
0 & 0 & 6 y(p) \\
0 & 6 y(p) & 0
\end{array}\right) .
$$

Then $M_{m}$ represents simultaneously the trace form in $O_{K_{m}}$ and $O_{L_{m}}$ with respect to the bases given by Proposition 3.3.

The pair of number fields given by Proposition 3.4 need not be isomorphic, as the following example demonstrates.

Example 3.5. Let $m=12$ so that $K_{12}$ and $L_{12}$ are the cubic fields defined by $x^{3}-12$ and $x^{3}-6$ respectively. Then $\left\langle O_{K}, \tilde{q}_{K_{12}}\right\rangle$ and $\left\langle O_{L}, \tilde{q}_{L_{12}}\right\rangle$ are isomorphic to $\left\langle\mathbb{Z}^{3}, 3 x^{2}+36 y z\right\rangle$. We see that $K_{12}$ and $L_{12}$ are nonisomorphic fields of discriminant $-2^{2} 3^{5}$ by direct computation; for instance, 7 splits in $L_{12}$ but is inert in $K_{12}$.

Recall that for Galois cubic fields of fixed discriminant, there is only one possibility for the trace form (see Theorem 3.1), since after a suitable scaling we are left with a binary quadratic form of discriminant -3 . Inspired by this, we began looking for discriminants $D$ of totally real cubic fields satisfying four conditions: (i) $D$ is a nonperfect square; (ii) $D$ is nonfundamental; (iii) up to square factors 
and factors of $3,-D$ defines an imaginary quadratic field of class number 1 . and (iv) there are at least two cubic fields of discriminant $D$.

It turns out that the first $D$ satisfying these conditions (see tables at the end of [Ennola and Turunen 1985]) is $D=66825=3^{5} 5^{2} 11$. For this value of $D$ we have:

Example 3.6. Let $K$ and $L$ be the cubic fields defined by $2 x^{3}+3 x^{2}-21 x+4$ and $x^{3}+9 x^{2}-18 x-3$ respectively. Then $\left\langle O_{K}, \tilde{q}_{K}\right\rangle$ and $\left\langle O_{L}, \tilde{q}_{L}\right\rangle$ are isomorphic to $\left\langle\mathbb{Z}^{3}, 3 x^{2}+90\left(y^{2}+y z+3 z^{2}\right)\right\rangle$. One sees by direct computation that $K$ and $L$ are nonisomorphic fields of discriminant $3^{5} 5^{2} 11$ (they have different regulators).

None of our results so far yield positive answers to Questions 1.1 or 1.3 with fundamental discriminant. It is thus natural to ask whether those questions have negative answers in the special case where the discriminant of the cubic field is fundamental. Under these circumstances we exhibit a more convenient refinement. To describe this, let $K$ be a cubic number field and recall our notation $q_{k}=\left.\tilde{q}_{K}\right|_{O_{K}^{0}}$. Then $q_{K}$ is an integral, binary quadratic form. Moreover, under the fundamental discriminant hypothesis, the isometry class of $\left\langle O_{K}^{0}, q_{K}\right\rangle$ is a refinement of the discriminant, as shown in Lemma 2.5.

\section{Cubic fields with fundamental discriminant}

In this section, all cubic fields are assumed to have fundamental discriminant. The first question that comes to mind is this: for which fundamental discriminants $d$ does there exist a cubic field with discriminant $d$ ? Moreover, we would like to know for which values of $d$ there is more than one isomorphism class of cubic fields of discriminant $d$. It turns out that class field theory gives nice answers to these questions. Let $K$ be a cubic field of fundamental discriminant $d$ and Galois closure $\widetilde{K}$. Clearly, $\mathbb{Q}(\sqrt{d}) \subseteq \widetilde{K}$, and this extension is unramified. Since $d$ is a fundamental discriminant, $\operatorname{Gal}(\widetilde{K} / \mathbb{Q}) \cong S_{3}$. Hence $[\widetilde{K}: \mathbb{Q}(\sqrt{d})]=3$, and $\widetilde{K} / \mathbb{Q}(\sqrt{d})$ is abelian. Therefore, if $H_{d}$ denotes the Hilbert class field of $\mathbb{Q}(\sqrt{d})$, and $\mathrm{Cl}_{\mathbb{Q}(\sqrt{d})}$ denotes the ideal class group of $\mathbb{Q}(\sqrt{d})$, we have this diagram:

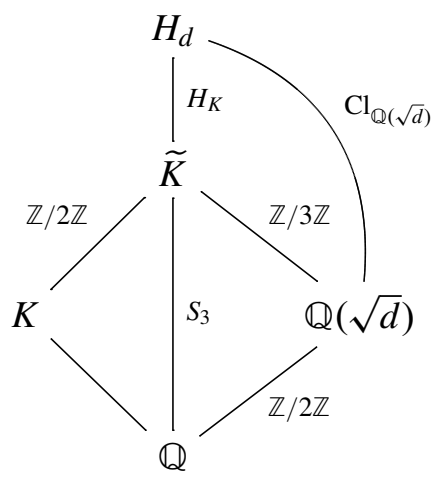


Thus, if we start with $K$ as above, we obtain $H_{K}$, an index-3 subgroup of $\mathrm{Cl}_{\mathbb{Q}(\sqrt{d})}$. Conversely, it can be shown [Hasse 1930] that the fixed field of an index-3 subgroup of $\mathrm{Cl}_{\mathbb{Q}(\sqrt{d})}$ corresponds to the Galois closure of a cubic field of discriminant $d$. Hence:

Proposition 4.1 [Hasse 1930]. The number of isomorphism classes of cubic fields of discriminant d is $\left(3^{\mathrm{r}_{3}(d)}-1\right) / 2$, where $\mathrm{r}_{3}(d)=\operatorname{dim}_{\mathbb{F}_{3}}\left(\mathrm{Cl}_{\mathbb{Q}(\sqrt{d})} \otimes_{\mathbb{Z}} \mathbb{F}_{3}\right)$.

Corollary 4.2 [Hasse 1930]. There exists a cubic field $K$ of discriminant $d$ if and only if $\mathrm{Cl}_{\mathbb{Q}(\sqrt{d})}[3] \neq 0$.

Section 3 gave affirmative answers to Questions 1.1 and 1.3 for nonfundamental discriminants. Example 4.3 shows that among fundamental discriminants, one still finds positive answers to Questions 1.1 and 1.3.

Example 4.3. The fundamental discriminant of least absolute value with $r_{3}(d)>1$ is $d=-3299$. For this value of $d, \mathrm{Cl}_{\mathbb{Q}(\sqrt{d})} \cong \mathbb{Z} / 3 \mathbb{Z} \oplus \mathbb{Z} / 9 \mathbb{Z}$; hence there exist four nonisomorphic cubic fields of discriminant -3299 . Among these four fields, the ones defined by $x^{3}+2 x+11$ and $x^{3}-16 x+27$ have isometric trace- 0 parts.

Cubic fields with square-free discriminants lead us to 3-torsion of class groups of quadratic fields. Another very well-known source of class groups of quadratic fields is binary quadratic forms. Let us recall briefly how these two are connected. Let $\Delta$ be a non-perfect-square integer and let $\Gamma_{\Delta}$ (respectively $\Gamma_{\Delta}^{1}$ ) be the set of $\mathrm{GL}_{2}(\mathbb{Z})$-equivalence classes (respectively $\mathrm{SL}_{2}(\mathbb{Z})$-equivalence classes) of primitive, binary quadratic forms of discriminant $\Delta$. Gauss composition gives a group structure to $\Gamma_{\Delta}^{1}$, and furthermore this group is isomorphic to the narrow class group $\mathrm{Cl}_{\mathbb{Q}(\sqrt{\Delta})}^{+}$. In particular, $\left|\Gamma_{\Delta}\right| \leq\left|\mathrm{Cl}_{\mathbb{Q}(\sqrt{\Delta})}^{+}\right|$. Now, let $K$ be a cubic field of discriminant $d$ not divisible by 3 . According to the next lemma, the $\mathrm{GL}_{2}(\mathbb{Z})$-equivalence class of $\left[\frac{1}{2} q_{K}\right]$ defines an element of $\Gamma_{-3 d}$. Thus, if we denote by $\mathscr{C}_{d}$ the set of isomorphism classes of cubic fields of discriminant $d$, we have the map

$$
\Phi_{d}: \mathscr{C}_{d} \rightarrow \Gamma_{-3 d}, \quad K \mapsto\left[\frac{1}{2} q_{K}\right] .
$$

Since $\mathrm{Cl}_{\mathbb{Q}(\sqrt{9897})}^{+} \cong \mathbb{Z} / 3 \mathbb{Z}$ and $\left|\mathscr{C}_{-3299}\right|=4$, the previous example can be restated as the noninjectivity of $\Phi_{-3299}$.

Lemma 4.4. Let $K$ be a cubic field with fundamental discriminant $d$. Then $\frac{1}{2} q_{K}$ is an integral, binary quadratic form of discriminant $-3 d$.

Proof. Note that $\operatorname{Disc} q_{K}=-4 \operatorname{Disc} O_{K}^{0}=-\frac{4}{3}\left|O_{K} / G_{K}\right|^{2} d$. Since $d$ is fundamental, $9 \nmid d$. In particular, $\operatorname{tr}_{K / \mathbb{Q}}$ is a surjection from $O_{K}$ to $\mathbb{Z}$, and thanks to Lemma 2.3, we have Disc $q_{K}=-12 d$. Note that if $x \in O_{K}^{0}$, then $\operatorname{tr}_{K / \mathbb{Q}}\left(x^{2}\right)=$ $\operatorname{tr}_{K / \mathbb{Q}}\left(x^{2}\right)-\operatorname{tr}_{K / \mathbb{Q}}^{2}(x) \in 2 \mathbb{Z}$, and hence $\frac{1}{2} q_{K}$ is integral.

Remark 4.5. In fact $\frac{1}{2} q_{K}$ is primitive if $3 \nmid d$, as seen in Corollary 5.4. 
Often it is more convenient to work with primitive forms than general ones.

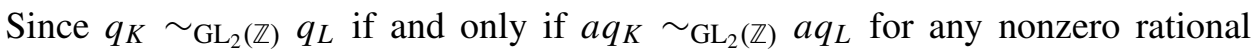
number $a$, Remark 4.5 will allow us to restrict ourselves to primitive forms.

\section{Trace form and class groups}

In this section we calculate $q_{K}$ explicitly, and then show that for positive fundamental discriminants, $q_{K}$ characterizes the field. We start by recalling [Delone and Faddeev 1964; Gan et al. 2002; Belabas and Cohen 1998] on the parametrization of cubic rings. Every conjugacy class of a cubic ring $R$ has associated to it a unique integral binary cubic form $(a, b, c, d):=F(x, y)=a x^{3}+b x^{2} y+c x y^{2}+d y^{3}$ up to $\mathrm{GL}_{2}(\mathbb{Z})$-equivalence. Let $K$ be a cubic number field and $F$ the form associated to its maximal order. Among the properties of $F$, we have:

- $K=\mathbb{Q}(\theta)$, where $\theta \in K$ is a root of $F_{K}(x, 1)$.

- $d_{K}:=\operatorname{Disc} K=\operatorname{Disc}(a, b, c, d)=b^{2} c^{2}-27 a^{2} d^{2}+18 a b c d-4 a c^{3}-4 b^{3} d$.

- The Hessian form of $F, H_{F}=(P, Q, R):=P x^{2}+Q x y+R y^{2}$, has discriminant $-3 d_{K}$, where

$$
P=b^{2}-3 a c, \quad Q=b c-9 a d, \quad R=c^{2}-3 b d .
$$

- $H_{F}$ is covariant with respect to the $\mathrm{GL}_{2}(\mathbb{Z})$-action on binary cubic forms and on binary quadratic forms.

- $\mathscr{B}=\{1,-a \theta, d / \theta\}$ is a $\mathbb{Z}$-basis of $O_{K}$.

- If $d_{K}$ is fundamental, then $H_{F}$ is a primitive, binary quadratic form.

Lemma 5.1. Let $\alpha=-a \theta$ and $\beta=d / \theta$. Then $H_{F}$ is realized as the integral quadratic form $\frac{3}{2} \operatorname{tr}_{K / \mathbb{Q}}\left(X^{2}\right)$ over the $\mathbb{Z}$-module

$$
O_{K}^{\mathscr{P}}=\operatorname{Span}_{\mathbb{Z}}\left\{\alpha-\frac{\operatorname{tr}_{K / \mathbb{Q}}(\alpha)}{3}, \beta-\frac{\operatorname{tr}_{K / \mathbb{Q}}(\beta)}{3}\right\} .
$$

Proof. Note that $a^{2} F(x / a, 1)$ and $d^{2} F(1, x / d)$ are the minimal polynomials over $\mathbb{Q}$ of $\alpha$ and $\beta$ respectively. Hence, $\operatorname{tr}_{K / \mathbb{Q}}(\alpha)=b, \operatorname{tr}_{K / \mathbb{Q}}(\beta)=-c, \operatorname{tr}_{K / \mathbb{Q}}(\alpha \beta)=$ $-3 a d, \operatorname{tr}_{K / \mathbb{Q}}\left(\alpha^{2}\right)=b^{2}-2 a c$, and $\operatorname{tr}_{K / \mathbb{Q}}\left(\beta^{2}\right)=c^{2}-2 b d$. From this and a simple calculation the result follows.

Proposition 5.2. Let $\alpha_{0}=\alpha-\frac{1}{3} \operatorname{tr}_{K / \mathbb{Q}}(\alpha)$ and $\beta_{0}=\beta-\frac{1}{3} \operatorname{tr}_{K / \mathbb{Q}}(\beta)$. Then

$$
O_{k}^{0}= \begin{cases}O_{1}=\operatorname{Span}_{\mathbb{Z}}\left\{\alpha_{0}, 3 \beta_{0}\right\} & \text { if } b \equiv 0 \quad(\bmod 3), \\ O_{2}=\operatorname{Span}_{\mathbb{Z}}\left\{3 \alpha_{0}, \beta_{0}\right\} & \text { if } c \equiv 0 \quad(\bmod 3), \\ O_{3}=\operatorname{Span}_{\mathbb{Z}}\left\{\alpha_{0}-\beta_{0}, 3 \beta_{0}\right\} & \text { if } b \equiv-c(\bmod 3), \\ O_{4}=\operatorname{Span}_{\mathbb{Z}}\left\{\alpha_{0}+\beta_{0}, 3 \beta_{0}\right\} & \text { if } b \equiv c \quad(\bmod 3) .\end{cases}
$$


Proof. By Lemma 5.1, $\left(\frac{3}{2} \operatorname{tr}_{K / \mathbb{Q}}\left(X^{2}\right) \mid O_{K}^{\mathscr{B}}\right)=-3 d_{K}$ or, equivalently,

$$
\left(\frac{1}{2} \operatorname{tr}_{K / \mathbb{Q}}\left(X^{2}\right) \mid O_{K}^{\Re}\right)=-\frac{1}{3} d_{K} \text {. }
$$

On the other hand,

$$
-3 d_{K}=\left(\frac{1}{2} \operatorname{tr}_{K / \mathbb{Q}}\left(X^{2}\right) \mid O_{K}^{0}\right)=\left[O_{K}^{\Re}: O_{K}^{0}\right]^{2}\left(\frac{1}{2} \operatorname{tr}_{K / \mathbb{Q}}\left(X^{2}\right) \mid O_{K}^{\mathscr{\Re}}\right) .
$$

It follows that $\left[O_{K}^{\mathscr{B}}: O_{K}^{0}\right]=3$. Notice that for each $i$, the given congruence conditions on $b$ and $c$ imply that $O_{i} \subseteq O_{K}^{0}$. Since $\left[O_{K}^{\mathscr{P}}: O_{i}\right]=3$ for $i \in\{1,2,3,4\}$, the result follows.

Corollary 5.3. Let $K$ be a cubic field and let $F_{K}=(a, b, c, d)$ be a cubic form associated to $K$. Let $H_{K}=(P, Q, R)$ be the Hessian of $F_{K}$. Then the binary quadratic form $\frac{1}{2} \operatorname{tr}_{K / \mathbb{Q}}\left(X^{2}\right)$ on the lattice $O_{K}^{0}$ can be explicitly described as follows:

$$
\begin{cases}(P / 3, Q, 3 R) & \text { if } b \equiv 0 \quad(\bmod 3), \\ (3 P, Q, R / 3) & \text { if } c \equiv 0 \quad(\bmod 3), \\ \left(3 P, 2 P-Q, \frac{1}{3}(P+R-Q)\right) & \text { if } b \equiv-c(\bmod 3), \\ \left(3 P, 2 P+Q, \frac{1}{3}(P+Q+R)\right) & \text { if } b \equiv c \quad(\bmod 3) .\end{cases}
$$

Proof. By Lemma 5.1, the matrix of $\frac{3}{2} \operatorname{tr}_{K / \mathbb{Q}}\left(X^{2}\right)$ over $O_{K}^{\mathscr{B}}$ in the basis $\left\{\alpha_{0}, \beta_{0}\right\}$ is given by

$$
M=\left(\begin{array}{cc}
P & Q / 2 \\
Q / 2 & R
\end{array}\right) .
$$

Let $N_{1}=\left(\begin{array}{ll}1 & 0 \\ 0 & 3\end{array}\right), N_{2}=\left(\begin{array}{ll}3 & 0 \\ 0 & 1\end{array}\right), N_{3}=\left(\begin{array}{lr}1 & -1 \\ 0 & 3\end{array}\right)$, and $N_{4}=\left(\begin{array}{rr}1 & 0 \\ -1 & 3\end{array}\right)$. Then the coordinates of the vector $N_{i}\left(\alpha_{0}, \beta_{0}\right)^{t}$ form a basis of $O_{i}$, for $i \in\{1,2,3,4\}$. Hence, $\frac{1}{3} N_{i} M N_{i}^{t}$ is the matrix that represents $\frac{1}{2} \operatorname{tr}_{K / \mathbb{Q}}\left(X^{2}\right)$ over $O_{i}$ in such a basis. After applying Proposition 5.2, the result follows.

From now on, whenever we choose a cubic form $F_{K}$ in the $\mathrm{GL}_{2}(\mathbb{Z})$-class given by the field $K$, what we mean by $\frac{1}{2} q_{K}$ is the quadratic form in the coordinates given by Corollary 5.3 .

Corollary 5.4. If $K$ is a cubic field with fundamental discriminant $d$ not divisible by 3 , then $\frac{1}{2} q_{K}$ is a primitive, integral, binary quadratic form of discriminant $-3 d$.

Proof. By Lemma 4.4, it remains only to prove that $\frac{1}{2} q_{K}$ is primitive. Since $H_{k}$ is primitive and $9 \nmid-3 d$, the result follows from Corollary 5.3.

For a fixed $F_{K}$ in the $\mathrm{GL}_{2}(\mathbb{Z})$-class given by the field $K$, we have found explicit relations between the binary quadratic forms $\frac{1}{2} q_{K}$ and $H_{K}$. Since they have the same discriminant, namely $-3 d_{K}$, one might ask what their relation is as elements of the group $\mathrm{Cl}_{\mathbb{Q}\left(\sqrt{-3 d_{K}}\right)}^{+}$. A small objection to this question is that even though $H_{K}$ represents a valid element of this group, $\frac{1}{2} q_{K}$ need not, since it may not be 
primitive. Yet, as Corollary 5.4 shows, $\frac{1}{2} q_{K}$ is primitive whenever 3 does not ramify in $K$. In this setting we are able to find the following connection between forms.

Theorem 5.5. Let $K$ be a cubic field with discriminant $d_{K}$. Assume that $d_{K}$ is fundamental and that 3 does not divide $d_{K}$. Let $F_{K}=(a, b, c, d)$ be a cubic in the $\mathrm{GL}_{2}(\mathbb{Z})$-equivalence class defined by $K$. Then $\frac{1}{2} q_{K} * C_{d_{K}}=H_{K}^{ \pm 1}$ as elements of $\mathrm{Cl}_{\mathbb{Q}\left(\sqrt{-3 d_{K}}\right)}^{+}$, where $C_{d_{K}}=\left(3,0, \frac{1}{4} d_{K}\right)$ or $C_{d_{K}}=\left(3,3, \frac{1}{4}\left(d_{K}+3\right)\right)$ in accordance with whether $d_{k} \equiv 0(\bmod 4)$ or $d_{k} \equiv 1(\bmod 4)$.

Proof. We work out the case when $d_{k} \equiv 1(\bmod 4)$, the other case being completely analogous. By Arndt's composition algorithm [Buell 1989, Theorem 4.10],

$$
\begin{cases}C_{K} *(P, Q, R)=(P / 3, Q, 3 R) & \text { if } b \equiv 0 \quad(\bmod 3), \\ C_{K} *(3 P, Q, R / 3)=(P, Q, R) & \text { if } c \equiv 0 \quad(\bmod 3), \\ C_{K} *(3 P, 2 P-Q,(P+R-Q) / 3)=(P, 2 P-Q, P+R-Q) & \text { if } b \equiv-c(\bmod 3), \\ C_{K} *(3 P, 2 P+Q,(P+Q+R) / 3)=(P, 2 P+Q, P+R+Q) & \text { if } b \equiv c \quad(\bmod 3) .\end{cases}
$$

Using the matrix $\left(\begin{array}{ll}1 & -1 \\ 0 & 1\end{array}\right)$, we see that we have the identities in $\mathrm{Cl}_{\mathbb{Q}}^{+}\left(\sqrt{-3 d_{K}}\right)$

$$
(P, 2 P-Q, P+R-Q)=H_{K}^{-1} \quad \text { and } \quad(P, 2 P+Q, P+R+Q)=H_{K} .
$$

Since $C_{K}$ is its own inverse, the result follows from the explicit description of $\frac{1}{2} q_{K}$ given in Corollary 5.3.

Remark 5.6. Note that given $K$, we have freedom in choosing $F_{K}$ in such a way that $b \not \equiv-c(\bmod 3)$. Hence Theorem 5.5 can be actually interpreted as saying that $\frac{1}{2} q_{K} * C_{d_{K}}=H_{K}$.

Remark 5.7. We denote the form $C_{K}$ by $C_{d_{K}}$ in order to stress the fact that this form only depends on the discriminant of $K$.

Bhargava's composition laws on cubes and their relation to the trace form. We have related the trace form, in the cubic case, to class groups of quadratic fields. There is a well-known generalization of Gauss's composition of quadratic forms to cubic forms. Inspired by this generalization, we expected some connection between the cubic forms attached to cubic number fields, and the quadratic forms given by the traces of these fields. We briefly recall some of the basics of Bhargava's laws on cubes and then we explain how to get such a connection (see Theorem 6.2).

In his Ph.D. thesis [2004], Bhargava generalizes the composition laws on binary quadratic forms of a fixed discriminant $\Delta$ discovered by Gauss. Bhargava defines a $\mathrm{SL}_{2}(\mathbb{Z}) \times \mathrm{SL}_{2}(\mathbb{Z}) \times \mathrm{SL}_{2}(\mathbb{Z})$-action on the set of $2 \times 2 \times 2$ integral cubes of discriminant $\Delta$. Let $\mathrm{Cl}\left(\mathbb{Z}^{2} \otimes \mathbb{Z}^{2} \otimes \mathbb{Z}^{2} ; \Delta\right)$ be the space of orbits given of action. Using 
the generalization of Gauss's composition mentioned above, Bhargava discovered a composition law on $\mathrm{Cl}\left(\mathbb{Z}^{2} \otimes \mathbb{Z}^{2} \otimes \mathbb{Z}^{2} ; \Delta\right)$.

In explicit terms, one can think of a $2 \times 2 \times 2$ integral cube $\mathscr{b}$ as a pair of $2 \times 2$ integral matrices $(A, B)$, where $A$ is the front face and $B$ is the back face. Let $Q_{1}(\mathscr{C})=$ $-\operatorname{det}(A x+B y), Q_{2}(\mathscr{C})=-\operatorname{det}\left(\left[\begin{array}{l}x \\ y\end{array}\right] \mid B\left[\begin{array}{l}x \\ y\end{array}\right]\right)$ and $Q_{3}(\mathscr{C})=-\operatorname{det}\left(A^{t}\left[\begin{array}{l}x \\ y\end{array}\right] \mid B^{t}\left[\begin{array}{l}x \\ y\end{array}\right]\right)$.

It can be verified that Disc $Q_{1}=\operatorname{Disc} Q_{2}=\operatorname{Disc} Q_{3}$. This common discriminant $\Delta$ is precisely the definition of the discriminant of $\mathscr{C}$. If

$$
g:=\left(g_{1}, g_{3}, g_{3}\right) \in \Gamma:=\mathrm{SL}_{2}(\mathbb{Z}) \times \mathrm{SL}_{2}(\mathbb{Z}) \times \mathrm{SL}_{2}(\mathbb{Z})
$$

and $(A, B)$ is a cube, then

$$
g \cdot(A, B):=g_{1}\left(\begin{array}{l}
g_{3} A g_{2}^{t} \\
g_{3} B g_{2}^{t}
\end{array}\right) .
$$

This action preserves the discriminant. Moreover, if $Q_{1}, Q_{2}, Q_{3}$ are primitive forms, one has that $Q_{1} * Q_{2} * Q_{3}=0$ as elements of $\mathrm{Cl}_{\mathbb{Q}(\sqrt{\Delta})}^{+}$. Conversely, let $\left(Q_{1}, Q_{2}, Q_{3}\right)$ be a triple of primitive, binary quadratic forms of discriminant $\Delta$ such that $Q_{1} * Q_{2} * Q_{3}=0$. Then there is a unique class on $\mathrm{Cl}\left(\mathbb{Z}^{2} \otimes \mathbb{Z}^{2} \otimes \mathbb{Z}^{2} ; \Delta\right)$ giving rise to $\left(Q_{1}, Q_{2}, Q_{3}\right)$ as above. With this in hand, it is simple to define a composition law on cubes: $(A, B)+\left(A^{\prime}, B^{\prime}\right)$ is the cube that corresponds to the triple $\left(Q_{1} * Q_{1}^{\prime}, Q_{2} * Q_{2}^{\prime}, Q_{3} * Q_{3}^{\prime}\right)$. Furthermore:

Theorem 5.8 [Bhargava 2004]. There is an isomorphism

$$
\phi: \mathrm{Cl}\left(\mathbb{Z}^{2} \otimes \mathbb{Z}^{2} \otimes \mathbb{Z}^{2} ; \Delta\right) \rightarrow \mathrm{Cl}_{\mathbb{Q}(\sqrt{\Delta})}^{+} \times \mathrm{Cl}_{\mathbb{Q}(\sqrt{\Delta})}^{+}
$$

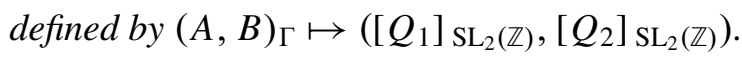

Definition 5.9. A binary cubic form $f(x, y) \in \mathbb{Z}[x, y]$ is called a Gaussian cubic form if it is of the form $\left(a_{0}, 3 a_{1}, 3 a_{2}, a_{3}\right)$. The set of Gaussian cubic forms is denoted by $\mathrm{Sym}^{3} \mathbb{Z}^{2}$.

One may naturally associate to a Gaussian cubic form $f=\left(a_{0}, 3 a_{1}, 3 a_{2}, a_{3}\right)$ a triple symmetric cube:

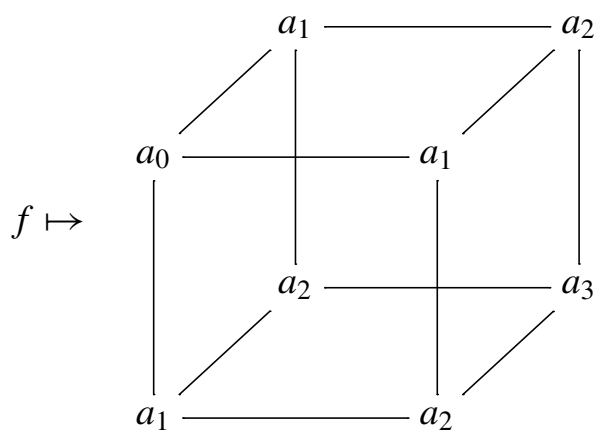


The correspondence between cubic forms and cubes is identified with a map

$$
\imath: \mathrm{Sym}^{3} \mathbb{Z}^{2} \rightarrow \mathbb{Z}^{2} \otimes \mathbb{Z}^{2} \otimes \mathbb{Z}^{2} .
$$

If we replace $f$ by a Gaussian form in the same $\mathrm{SL}_{2}(\mathbb{Z})$ equivalence class as $f$, one obtains a well defined element under the $\Gamma$-action on cubes.

Let

$$
\mathrm{Cl}\left(\mathrm{Sym}^{3} \mathbb{Z}^{2} ; \Delta\right)
$$

be the set of Gaussian forms, up to $\mathrm{SL}_{2}(\mathbb{Z})$-action, such that the corresponding cubes have fundamental discriminant $\Delta$.

Remark 5.10. One must distinguish between the notions of the discriminant of cubic forms and the discriminant of cubes. For example, let $f$ be a Gaussian form of discriminant $D$. Then the cube corresponding to $f$ has discriminant $\Delta=-\frac{1}{27} D$.

It turns out that $\mathrm{Cl}\left(\mathrm{Sym}^{3} \mathbb{Z}^{2} ; \Delta\right)$ is an abelian group. Furthermore,

$$
[l]:[f]_{\mathrm{SL}_{2}(\mathbb{Z})} \mapsto[l(f)]_{\Gamma}
$$

is a group homomorphism. By composing the homomorphisms

$$
\mathrm{Cl}\left(\operatorname{Sym}^{3} \mathbb{Z}^{2} ; \Delta\right) \stackrel{[l]}{\rightarrow} \mathrm{Cl}\left(\mathbb{Z}^{2} \otimes \mathbb{Z}^{2} \otimes \mathbb{Z}^{2} ; \Delta\right) \stackrel{\phi}{\rightarrow} \mathrm{Cl}_{\mathbb{Q}(\sqrt{\Delta})}^{+} \times \mathrm{Cl}_{\mathbb{Q}(\sqrt{\Delta})}^{+} \stackrel{\pi_{1}}{\rightarrow} \mathrm{Cl}_{\mathbb{Q}(\sqrt{\Delta})}^{+},
$$

Bhargava obtains:

Theorem 5.11 [Bhargava 2004; Hoffman and Morales 2000]. There is a surjective homomorphism

$$
\phi_{1}: \mathrm{Cl}\left(\operatorname{Sym}^{3} \mathbb{Z}^{2} ; \Delta\right) \rightarrow \mathrm{Cl}_{\mathbb{Q}(\sqrt{\Delta})}^{+}[3],
$$

where $\phi_{1}$ is the first projection of $\phi$ composed with $[\mathrm{l}]$. The cardinality of the kernel is equal to $\left|U / U^{3}\right|$, where $U$ denotes the group of units in $\mathbb{Q}(\sqrt{\Delta})$. In other words, the kernel has order 1 if $\Delta<-3$, or 3 otherwise.

This theorem was in essence first obtained by Eisenstein [1844], but he incorrectly asserted that the kernel of the map was always trivial. Later Arnt and Cayley pointed out that it is not a bijection if $\Delta \geq-3$.

Remark 5.12. Explicitly,

$$
\phi_{1}\left(a_{0}, 3 a_{1}, 3 a_{2}, a_{3}\right)=\left(a_{1}^{2}-a_{0} a_{2}, a_{1} a_{2}-a_{0} a_{3}, a_{2}^{2}-a_{1} a_{3}\right) .
$$

\section{From cubic fields to cubes and trace forms}

Given $K$, a cubic field of discriminant $d_{K}$, and representative form $F_{K}(x, y)=$ $(a, b, c, d)$, we naturally associate a cube as follows: 
$K: a x^{3}+b x^{2} y+c x y^{2}+d y^{3} \longmapsto$

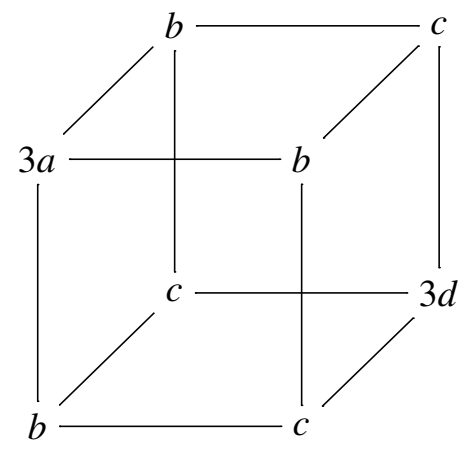

We obtain in this way an element

$$
\mathscr{K}_{F} \in[\imath]\left(\mathrm{Cl}\left(\mathrm{Sym}^{3} \mathbb{Z}^{2} ;-3 d_{K}\right)\right) \subseteq \mathrm{Cl}\left(\mathbb{Z}^{2} \otimes \mathbb{Z}^{2} \otimes \mathbb{Z}^{2} ;-3 d_{K}\right) .
$$

Let $D$ be a fundamental discriminant. Let $\mathscr{C}_{D} \in \mathrm{Cl}\left(\mathbb{Z}^{2} \otimes \mathbb{Z}^{2} \otimes \mathbb{Z}^{2} ;-3 D\right)$ be given by
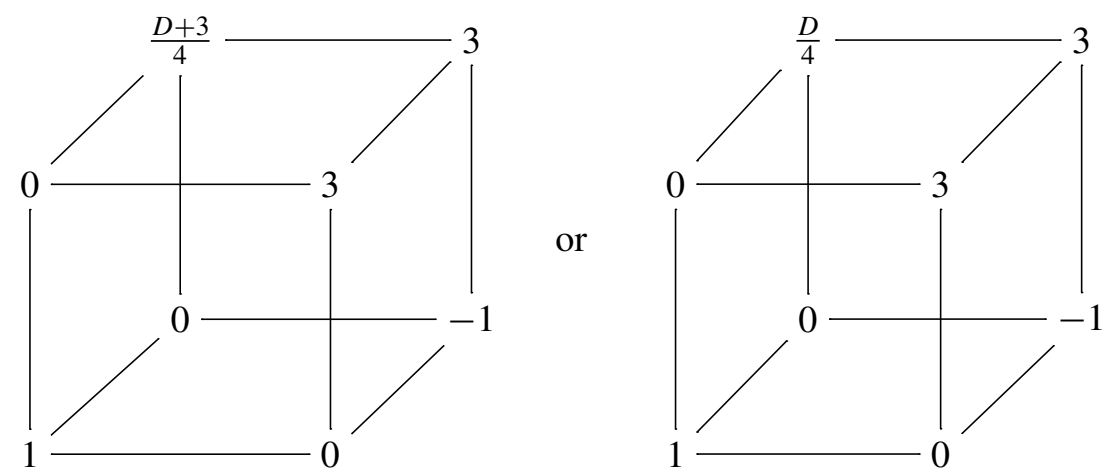

in accordance with whether $D \equiv 0(\bmod 4)$ or $D \equiv 1(\bmod 4)$.

Lemma 6.1. Let $K$ be a cubic field with a fixed cubic form $F=(a, b, c, d)$. Then $Q_{1}\left(\mathscr{K}_{F}\right)=H_{F}$ and $Q_{1}\left(\mathscr{C}_{d_{K}}\right)=C_{d_{K}}$.

Proof. The result follows easily using the definition $Q_{1}(A, B)=-\operatorname{det}(A x+B y)$ for a cube $(A, B)$.

Theorem 6.2. Let $K$ be a cubic field with discriminant $d_{K}$ and associated cubic form $F_{K}=(a, b, c, d)$. Assume that $d_{K}$ is fundamental and that 3 does not ramify. Let $T_{F_{K}}=\mathscr{K}_{F}+\mathscr{C}_{d_{K}}$. Then $\left(\pi_{1} \circ \phi\right)\left(T_{F_{K}}\right)^{ \pm 1}=\frac{1}{2} q_{K}$ as elements of $\mathrm{Cl}_{\mathbb{Q}\left(\sqrt{-3 d_{K}}\right.}^{+}$.

Proof. Since $\phi$ is a group homomorphism, we have

$$
\phi\left(T_{F_{K}}\right)=\phi\left((K)_{F}\right) * \phi\left(\mathscr{C}_{d_{K}}\right) .
$$

Projecting to the first component by $\pi_{1}$, we get that $\left(\pi_{1} \circ \phi\right)\left(T_{F_{K}}\right)=H_{K} * C_{d_{K}}$. Since all of the functions involved are group homomorphisms, the result follows 
from Theorem 5.5. In other "words",
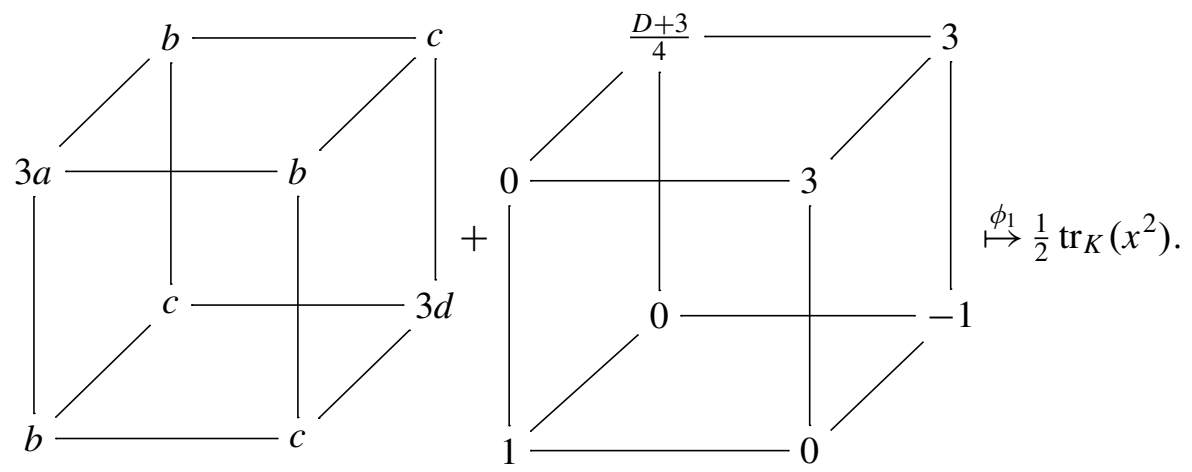

Remark 6.3. We could choose $F_{K}$ (see Remark 5.6) so that the conclusion of Theorem 6.2 is $\left(\pi_{1} \circ \phi\right)\left(T_{F_{K}}\right)=\frac{1}{2} q_{K}$.

Theorem 6.4. Let $K$ be a cubic field with discriminant $d_{K}$, and let $F_{K}(x, y)=$ $(a, b, c, d)$ be a cubic form associated to $K$. Assume that $d_{K}$ is fundamental and that 3 ramifies in $K / \mathbb{Q}$. Then we have

$$
\begin{aligned}
& \phi_{1}: \mathrm{Cl}\left(\mathrm{Sym}^{3} \mathbb{Z}^{2} ;-d_{K} / 3\right) \rightarrow \mathrm{Cl}_{\mathbb{Q}\left(\sqrt{-d_{K} / 3}\right)}^{+}[3] \\
&\left(f_{K}\right)_{\mathrm{SL}_{2}(\mathbb{Z})} \mapsto\left(\frac{1}{6} q_{K}\right) \mathrm{SL}_{2}(\mathbb{Z}),
\end{aligned}
$$

where

$$
f_{K}(x, y):= \begin{cases}\frac{1}{3} F(x, 3 y) & \text { if } b \equiv 0 \quad(\bmod 3), \\ \frac{1}{3} F(3 x, y) & \text { if } c \equiv 0 \quad(\bmod 3), \\ \frac{1}{3} F(x, 3(y-x)) & \text { if } b \equiv-c(\bmod 3), \\ \frac{1}{3} F(x, 3(y+x)) & \text { if } b \equiv c \quad(\bmod 3) .\end{cases}
$$

Proof. Replacing $F(x, y)$ with either $F(y, x), F(x, y-x)$ or $F(x, y+x)$, we may assume that $b \equiv 0(\bmod 3)$. With this in hand, we have that $d_{K} \equiv-a c^{3}(\bmod 3)$, and since 3 ramifies, $a c \equiv 0(\bmod 3)$. On the other hand, since $d_{K}$ is fundamental, we see that $3 \mid a$. By Corollary 5.3, $\frac{1}{2} q_{K}=\left(\left(b^{2}-3 a c\right) / 3, b c-9 a d, 3\left(c^{2}-3 b d\right)\right)$, and thus $\frac{1}{6} q_{K}=\left(\left(\frac{1}{3} b\right)^{2}-\frac{1}{3} a c, \frac{1}{3} b c-\frac{a}{3} 9 d,\left(c^{2}-\frac{b}{3} 9 d\right)\right)$, which is $\phi_{1}\left(\frac{1}{3} F(x, 3 y)\right)$.

Theorem 6.5. Let $K$ be a cubic number field of positive, fundamental discriminant, and let $L$ be a number field such there exists an isomorphism of quadratic modules

$$
\left\langle O_{K}^{0}, q_{K}\right\rangle \cong\left\langle O_{L}^{0}, q_{L}\right\rangle .
$$

Further assume $9 \nmid d_{L}$. Then $K \cong L$.

Proof. By Lemma 2.5, we have $d_{K}=d_{L}$. As usual, fix cubic forms $F_{K}(x, y)$ and $F_{L}(x, y)$ in the classes given by $K$ and $L$ respectively. Suppose first that $3 \nmid d_{K}$. 
Since the isometry between the forms need not be proper, we can only ensure that, as elements of $\mathrm{Cl}_{\mathbb{Q}\left(\sqrt{-3 d_{K}}\right)}^{+}$,

$$
\frac{1}{2} q_{K}=\left(\frac{1}{2} q_{L}\right)^{ \pm 1} .
$$

By Theorem 6.2, we have $\left(\pi_{1} \circ \phi\right)\left(T_{F_{K}}\right)^{ \pm 1}=\left(\pi_{1} \circ \phi\right)\left(T_{F_{L}}\right)$. Replacing $F_{K}(x, y)$ by $F_{K}(x,-y)$ has the effect of replacing $H_{F_{K}}(x, y)$ by $H_{F_{K}}(x,-y)$. On the other hand, $H_{F_{K}}(x,-y)$ is inverse to $H_{F_{K}}$ in the narrow class group. Since $C_{d_{K}}$ has order 2 , Theorem 5.5 says that we may replace $F_{K}(x, y)$ by $F_{K}(x,-y)$, if necessary, so we may assume that

$$
\left(\pi_{1} \circ \phi\right)\left(T_{F_{K}}\right)=\left(\pi_{1} \circ \phi\right)\left(T_{F_{L}}\right)
$$

Equivalently,

$$
\left(\pi_{1} \circ \phi\right)\left(\mathscr{K}_{F_{K}}\right)=\left(\pi_{1} \circ \phi\right)\left(\mathscr{K}_{F_{L}}\right) .
$$

Notice that $\mathscr{K}_{F}=\imath(3 F)$, so $\phi_{1}\left(3 F_{K}\right)=\phi_{1}\left(3 F_{L}\right)$. Since $d_{K}>1$, Theorem 5.11 implies that $3 F_{K}$ and $3 F_{L}$ are $\mathrm{SL}_{2}(\mathbb{Z})$-equivalent. Since we could have replaced $F_{K}(x, y)$ by $F_{K}(x,-y)$, the equivalence between $3 F_{K}$ and $3 F_{L}$ is up to $\mathrm{GL}_{2}(\mathbb{Z})$. In any case this implies that $K \cong L$. If $3 \mid d_{K}$, we apply Theorem 6.4 and the argument follows the same lines as in the case without 3-ramification.

Observations. Given $\Delta \in \mathbb{Z}$, let $X_{\Delta}$ be the set of integral, primitive, binary quadratic forms of discriminant $\Delta$. Recall our notation $\Gamma_{\Delta}=\mathrm{GL}_{2}(\mathbb{Z}) \backslash X_{\Delta}$ and $\Gamma_{\Delta}^{1}=\mathrm{SL}_{2}(\mathbb{Z}) \backslash X_{\Delta}$.

Let $d$ be a positive fundamental discriminant, $n_{d}:=\operatorname{gcd}(3, d)$, and $\mathscr{C}_{d}$ the set of isomorphism classes of cubic fields of discriminant $d$.

Remark 6.6. Theorem 6.5 is equivalent to the injectivity of

$$
\Phi_{d}: \mathscr{C}_{d} \rightarrow \Gamma_{-3 d / n_{d}^{2}}, \quad K \mapsto\left[\frac{1}{2 n_{d}} q_{K}\right] .
$$

Since Gauss's composition induces a group isomorphism between $\mathrm{Cl}_{\mathbb{Q}}^{+}\left(\sqrt{-3 d / n_{d}^{2}}\right)$ and $\Gamma_{-3 d / n_{d}^{2}}^{1}$, we have a double cover

$$
\pi: \mathrm{Cl}_{\mathbb{Q}}^{+}\left(\sqrt{-3 d / n_{d}^{2}}\right) \rightarrow \Gamma_{-3 d / n_{d}^{2}}
$$

with the property that the fiber of every point consists of an element and its inverse. Therefore, even though $q_{K} / 2 n_{d}$ does not define a point in $\mathrm{Cl}_{\mathbb{Q}}^{+}\left(\sqrt{-3 d / n_{d}^{2}}\right)$, it defines a cyclic subgroup, the one generated by $\pi^{-1}\left(\Phi_{d}(K)\right)$. Corollary 5.3 and Theorem 6.4 provide us with a generator of this group. Let $g_{k}$ be such a generator. Using Arndt's composition algorithm [Buell 1989], one sees that $g_{K}^{3}=C_{K}$ when $3 \nmid d$, and that $g_{K}$ has order 3 otherwise. Since $C_{d_{K}}$ has order 2, it follows that $\left\langle\pi^{-1}\left(\Phi_{d}(K)\right)\right\rangle$ has order $2 n_{d}$.

Proposition 6.7. Let $d>0$ be a fundamental discriminant. The map $K \mapsto\left\langle g_{K}\right\rangle$ is injective. 
Proof. Since $\left\langle g_{K}\right\rangle$ has order 3 or 6 , its set of generators is $\left\{g_{K}^{ \pm 1}\right\}$. Thus, if $\left\langle g_{K}\right\rangle=\left\langle g_{L}\right\rangle$, then $g_{K}^{ \pm 1}=g_{L}$. Projecting under $\pi$, we obtain $\Phi_{d}(K)=\Phi_{d}(L)$, and the result follows from Remark 6.6.

The unique subgroup of order 3 of $\left\langle g_{K}\right\rangle$ is given by $\left\langle g_{K}^{2}\right\rangle$. From Proposition 6.7 we thus have:

Theorem 6.8. Let $d>0$ be a fundamental discriminant such that $\mathscr{C}_{d} \neq \varnothing$. Let $\mathscr{P}_{3}\left(\mathrm{Cl}_{\mathbb{Q}(\sqrt{-3 d})}^{+}\right)$be the set of subgroups of size 3 of $\mathrm{Cl}_{\mathbb{Q}(\sqrt{-3 d})}^{+}$. Then

$$
\Theta_{d}: \mathscr{C}_{d} \rightarrow \mathscr{P}_{3}\left(\mathrm{Cl}_{\mathbb{Q}(\sqrt{-3 d})}\right), \quad K \mapsto\left\langle g_{K}^{2}\right\rangle
$$

is injective.

The injection $\Theta_{d}$ provides an alternative proof for one inequality of the Scholz reflection principle [1932].

Corollary 6.9. Let $d$ be a positive fundamental discriminant, and let $r=\mathrm{r}_{3}(-3 d)$ and $s=\mathrm{r}_{3}(d)$ (recall our notation $\mathrm{r}_{3}(d)=\operatorname{dim}_{\mathbb{F}_{3}}\left(\mathrm{Cl}_{\mathbb{Q}(\sqrt{d})} \otimes_{\mathbb{Z}} \mathbb{F}_{3}\right)$ ). Then $s \leq r$. Proof. $\left(3^{s}-1\right) / 2=\left|\mathscr{C}_{d}\right|$ and $\left(3^{r}-1\right) / 2=\left|\mathscr{P}_{3}\left(\mathrm{Cl}_{\mathbb{Q}(\sqrt{-3 d})}\right)\right|$.

\section{Acknowledgements}

I thank Jordan Ellenberg for introducing me to this subject, and for many helpful discussions and suggestions during the writing of this paper. I also thank Manjul Bhargava, Amanda Folsom, and Yongqiang Zhao for thorough and helpful comments on an earlier version of this paper.

\section{References}

[Belabas and Cohen 1998] K. Belabas and H. Cohen, "Binary cubic forms and cubic number fields", pp. 191-219 in Computational perspectives on number theory (Chicago, 1995), edited by D. A. Buell and J. T. Teitelbaum, AMS/IP Stud. Adv. Math. 7, Amer. Math. Soc., Providence, 1998. MR 98m:11027 Zbl 0915.11024

[Bhargava 2004] M. Bhargava, "Higher composition laws, I: A new view on Gauss composition, and quadratic generalizations", Ann. of Math. (2) 159:1 (2004), 217-250. MR 2005f:11062a

[Buell 1989] D. A. Buell, Binary quadratic forms, Springer, New York, 1989. MR 92b:11021 Zbl 0698.10013

[Conner and Perlis 1984] P. E. Conner and R. Perlis, A survey of trace forms of algebraic number fields, Series in Pure Mathematics 2, World Scientific, Singapore, 1984. MR 86g:11021 Zbl 0551. 10017

[Delone and Faddeev 1964] B. N. Delone and D. K. Faddeev, The theory of irrationalities of the third degree, Translations of Mathematical Monographs 10, Amer. Math. Soc., Providence, 1964. MR 28 \#3955 Zbl 0133.30202

[Eisenstein 1844] G. Eisenstein, “Théorèmes sur les formes cubiques et solution d'une équation du quatrième degré à quatre indéterminées”, J. Reine Angew. Math. 27 (1844), 75-79. 
[Ennola and Turunen 1985] V. Ennola and R. Turunen, "On totally real cubic fields", Math. Comp. 44:170 (1985), 495-518. MR 86e:11100 Zbl 0564.12006

[Gan et al. 2002] W. T. Gan, B. Gross, and G. Savin, "Fourier coefficients of modular forms on $G_{2}$ ", Duke Math. J. 115:1 (2002), 105-169. MR 2004a:11036 Zbl 1165.11315

[Hasse 1930] H. Hasse, "Arithmetische Theorie der kubischen Zahlkörper auf klassenkörpertheoretischer Grundlage", Math. Z. 31:1 (1930), 565-582. MR 1545136

[Hilbert 1900] D. Hilbert, “Theorie der algebraischen Zahlkörper”, Encykl. d. math. Wiss. 1 (1900), 675-714. JFM 31.0207.01

[Hoffman and Morales 2000] J. W. Hoffman and J. Morales, "Arithmetic of binary cubic forms", Enseign. Math. (2) 46:1-2 (2000), 61-94. MR 2001h:11048 Zbl 0999.11021

[Marcus 1977] D. A. Marcus, Number fields, Springer, New York, 1977. MR 56 \#15601 Zbl 0383. $12001 \mathrm{Zbl}$

[Scholz 1932] A. Scholz, "Über die Beziehung der Klassenzahlen quadratischer Körper zueinander", J. Reine Angew. Math. 166 (1932), 201-203. Zbl 0004.05104

Communicated by Raman Parimala

Received 2009-06-18 Revised 2009-12-05 Accepted 2010-05-15

mantilla@math.ubc.ca Department of Mathematics,

University of Wisconsin-Madison, 480 Lincoln Drive, Madison, WI 53705, United States 


\section{Algebra \& Number Theory}

www.jant.org

\section{EDITORS}

\section{MANAGING EDITOR}

Bjorn Poonen

Massachusetts Institute of Technology

Cambridge, USA

\author{
EDITORIAL BOARD CHAIR \\ David Eisenbud \\ University of California \\ Berkeley, USA
}

\section{BOARD OF EDITORS}

\section{Georgia Benkart}

Dave Benson

Richard E. Borcherds

John H. Coates

J-L. Colliot-Thélène

Brian D. Conrad

Hélène Esnault

Hubert Flenner

Edward Frenkel

Andrew Granville

Joseph Gubeladze

Ehud Hrushovski

Craig Huneke

Mikhail Kapranov

Yujiro Kawamata

János Kollár

Hendrik W. Lenstra

Yuri Manin

Barry Mazur
University of Wisconsin, Madison, USA

University of Aberdeen, Scotland

University of California, Berkeley, USA

University of Cambridge, UK

CNRS, Université Paris-Sud, France

University of Michigan, USA

Universität Duisburg-Essen, Germany

Ruhr-Universität, Germany

University of California, Berkeley, USA

Université de Montréal, Canada

San Francisco State University, USA

Hebrew University, Israel

University of Kansas, USA

Yale University, USA

University of Tokyo, Japan

Princeton University, USA

Universiteit Leiden, The Netherlands

Northwestern University, USA

Harvard University, USA
Susan Montgomery

Shigefumi Mori

Andrei Okounkov

Raman Parimala

Victor Reiner

Karl Rubin

Peter Sarnak

Michael Singer

Ronald Solomon

Vasudevan Srinivas

J. Toby Stafford

Bernd Sturmfels

Richard Taylor

Ravi Vakil

Michel van den Bergh

Marie-France Vignéras

Kei-Ichi Watanabe

Andrei Zelevinsky

Efim Zelmanov
University of Southern California, USA

RIMS, Kyoto University, Japan

Princeton University, USA

Emory University, USA

University of Minnesota, USA

University of California, Irvine, USA

Princeton University, USA

North Carolina State University, USA

Ohio State University, USA

Tata Inst. of Fund. Research, India

University of Michigan, USA

University of California, Berkeley, USA

Harvard University, USA

Stanford University, USA

Hasselt University, Belgium

Université Paris VII, France

Nihon University, Japan

Northeastern University, USA

University of California, San Diego, USA

\section{PRODUCTION}

ant@mathscipub.org

Silvio Levy, Scientific Editor

Andrew Levy, Production Editor

See inside back cover or www.jant.org for submission instructions.

The subscription price for 2010 is US \$140/year for the electronic version, and \$200/year (+\$30 shipping outside the US) for print and electronic. Subscriptions, requests for back issues from the last three years and changes of subscribers address should be sent to Mathematical Sciences Publishers, Department of Mathematics, University of California, Berkeley, CA 94720-3840, USA.

Algebra \& Number Theory (ISSN 1937-0652) at Mathematical Sciences Publishers, Department of Mathematics, University of California, Berkeley, CA 94720-3840 is published continuously online. Periodical rate postage paid at Berkeley, CA 94704, and additional mailing offices.

ANT peer review and production are managed by EditFLOW ${ }^{\mathrm{TM}}$ from Mathematical Sciences Publishers.

\section{PUBLISHED BY}

mathematical sciences publishers

http://www.mathscipub.org

A NON-PROFIT CORPORATION

Typeset in LATEX

Copyright $\odot 2010$ by Mathematical Sciences Publishers 


\section{Algebra \& Number Theory}

Volume $4 \quad$ No. $6 \quad 2010$

Generalized moonshine I: Genus-zero functions

SCOTT CARNAHAN

Integral trace forms associated to cubic extensions

GUILLERMO MANTILLA-SOLER

RACHEL OLLIVIER

DANNY NEFTIN and ELAD PARAN

John E. Cremona, Tom A. Fisher and Michael Stoll 